\title{
Omega-3 Fatty Acids-Related Cancer Prevention: An Opinion
}

\author{
Nathalia Pizato* \\ Department of Nutrition, Post Graduate Program in Human Nutrition, Brazil
}

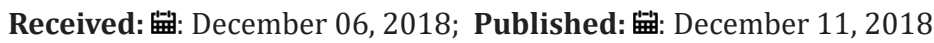

*Corresponding author: Nathalia Pizato, Department of Nutrition, Post Graduate Program in Human Nutrition, Brazil

Keywords: Fatty Acids; Omega 3; Cancer

\section{Opinion}

Fatty acids are key components of phospholipid membranes and lipid are related to cell signaling thought to impact carcinogenesis process. Multiple mechanisms are related to this chemopreventive activity, including cell growth inhibition, control of cell cycle arrest and suppression of neoplastic transformation. Omega 3 (n3) polyunsaturated fatty acids (PUFA) are found naturally in fish or fish oil and have been extensively studied in multiple inflammatory diseases with improved outcomes, especially in cancer, whereas $n 3$ PUFA appear to modulate cancer progression while $n 6$ fatty acids contribute to cancer development.

Major changes have taken place in the food supply over the last 100 years, which lead to increasing the amount of $n 6$ fatty acids intake from vegetable oils and meat, eggs, dairy, associated with fish consumption decreased, known as Western Diet. This food intake pattern led to a n6/n3 ratio imbalance intake for the first time in the history of human beings since industrial revolution. An increased incidence of cancer in western societies is associated with low ratios of omega-3: omega- 6 fatty acid intake [1].

With a higher $\mathrm{n} 3$ intake or supplementation, the replacement of $n 6$ fatty acids in cell membranes by $n 3$ fatty acids is also an important anti-cancer mechanism of action. Incorporation of $\mathrm{n} 3$ fatty acids in the cell membrane corrects abnormal cellular proliferation and decrease inflammation-associated carcinogenesis. Thus, they can directly influence cancer cells and the tumor environment and also influence the host response to tumor bearing progression. Today, US Department of Agriculture and World Health Organization recommends to healthy adults a higher intake of w3 long chain polyunsaturated fatty acids, such as eicosapentaenoic acid (EPA) and docosahexaenoic acid (DHA). But given the level of omega- 6 fatty acids in Western diets an intake of 2 to $3 \mathrm{~g} /$ day combined EPA and DHA, or at least $2 \%$ of total calories, is likely to show better physiologic effects.

Because of the increased amounts of n 6 fatty acids in the Western diet, their inflammatory eicosanoid metabolic products, specifically prostaglandins, thromboxanes and leukotrienes are formed in larger quantities than those formed from $n 3$ fatty acids. The eicosanoids from $\mathrm{n} 6$ are more biologically active than $\mathrm{n} 3$ ones and if they are formed in large amounts, they contribute to promote tumor cell proliferation, survival, migration and invasion [2]. The mechanisms include alteration in membrane lipid rafts, which modifies pro-inflammatory lipid eicosanoid cited above, the nuclear factor- $\kappa \mathrm{B}-\mathrm{in}$ duced cytokine production inhibition and decreased growth factor receptor activity.

Another mechanism is that nuclear factor- $\mathrm{\kappa B}$ nuclear translocation and signaling is reduced via the agonist effects of EPA and DHA on a specific receptor called peroxisome proliferator-activated receptor gamma (PPAR gamma) reducing inflammation and cancer progressiveness. The interactions of EPA and DHA also occurs with the G protein receptor GPR120, with expected reduction in inhibitors of apoptosis as well as cytokines, adhesion molecules, and metalloproteases, and other proteins associated with cell cycle control and DNA repair.

In experimental animals and cell culture studies the cancer prevent effects of w3 fatty acids are pacified. However, in human studies there are still no concluded evidences. Systematic reviews of epidemiologic and clinical studies have showed that colorectal, prostate and breast cancers provided limited evidence of a possible role of n3 PUFA in cancer prevention. The explanation is due to the methodological heterogeneity of the observations. Confounding factors as dietary pattern context, level of PUFA intake, time of sup- 
plementation and genetic polymorphism could explain the difficulties associated with human studies.

Another important issue about PUFAS are their clinical use as adjuvant therapy for cancer. Weight loss is very common during clinical treatment and the anti-inflammatory effects of EPA and DHA might help reduce muscle mass loss and free fat mass, favoring weight gain during treatment, but more studies in this area have yet to be conducted to make this association clearer. In an interesting review of the literature Vaughan et al. [3] concluded "... the use of supplements containing $\mathrm{n} 3$ fatty acids may have potential use as an effective adjuvant to chemotherapy treatment and may help ameliorate some of the secondary complications associated with cancer..." Thus, examination of the interactions of omega 3 FAs intake or supplements with well-known anticancer drugs, could provide better human clinical outcomes.

Due to the different genetic background and tumor subtypes, type of dietary sources used, fatty acid bioavailability there is still no consensus about fatty acids doses supplementation and length

\section{ISSN: 2574-1241}

DOI: $10.26717 / B J S T R .2018 .11 .002178$

Nathalia Pizato. Biomed J Sci \& Tech Res

(C) (i) This work is licensed under Creative

Submission Link: https://biomedres.us/submit-manuscript.php of treatment in cancer patients. On the other hand, n- 6 fatty acids are often associated with a higher cancer risk, even if their beneficial effects have also been highlighted in some experimental studies.

In conclusion, it is extremely important to continue the discussion about the dietary source, bioavailability, biochemistry and metabolism of omega 3 fatty acids, their potential anticancer molecular mechanisms and possible adverse effects, to guarantee the effectiveness in cancer prevention and treatment in human.

\section{References}

1. Simopoulos AP (2011) Importance of the omega-6/omega-3 balance in health and disease: Evolutionary aspects of diet. World Rev Nutr Diet 102: 10-21.

2. Innes JK, Calder PC (2018) Omega-6 fatty acids and inflammation. Prostaglandins Leukot Essent Fatty Acids 132: 41-48.

3. Vaughan VC, Hassing MR, Lewandowski PA (2013) Marine polyunsaturated fatty acids and cancer therapy. Brit J Cancer 108(3): 486-492.

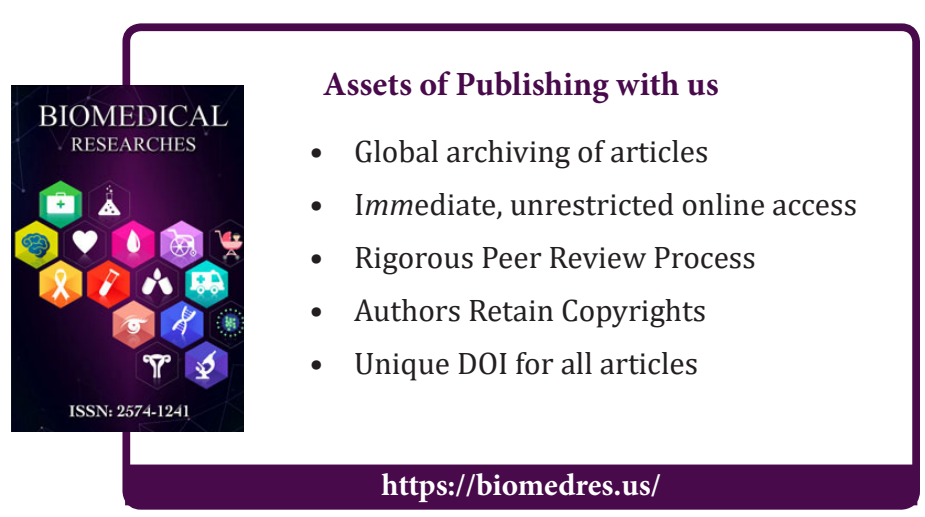

